\title{
The morphologic changes in the wall of the human urinary tracts after direct electro pulse exposure: the in vitro research
}

\author{
A.V. Gudkov, Vyacheslav S. Boshchenko, V.V. Nedosekov
}

Siberian State Medical University, Tomsk, Russia

Accepted 21 January 2013

Original Text in Russian () Gudkov AV, Boshchenko VS, Nedosekov VV, 2011, published in Saratov Journal of Medical Scientific Research 2011; 7(4): 937-941.

\begin{abstract}
The aim - study of the morphological changes in the wall of the human urinary tracts, developing after direct electro pulse exposure (DEPE). Materials and methods - The fragments of the surgical material obtained after the pelvis $(n=25)$, ureter $(n=33)$ and urinary bladder $(n=20)$ open operations, were used in the research. DEPE was carried out by single (from 1 to 20) nanosecond pulses with the power of $0.8 \mathrm{~J}$ (joule) and $1.0 \mathrm{~J}$ made by the electro pulse "Urolit-105M" lithotriptor probe on the fragments' mucous tunic placed in the Petri dishes with $0.9 \% \mathrm{NaCl}$ solution. The fragments were fixed in the formalin and after the processing, and pouring with paraffin, the tissue slices were prepared. Results - The kidney pelvis wall, where changes like epithelium desquamation, the basal membrane disorganization, paretic capillary expansion did not go beyond sub mucous layer even after exposure by 10 pulses with the power $1.0 \mathrm{~J}$ each, aimed at one point, proved to be the most resistant to DEPE. In the fragments of ureter and urinary bladder the same changes were detected after exposure by 5 pulses with the power of $1.0 \mathrm{~J}$ each, whereas after exposure by 10 pulses with the power $1.0 \mathrm{~J}$ each the changes occurred which were restricted by the muscular layer. Conclusion - The DEPEon the mucous tunic of the isolated urinary tracts fragments by the means of 10 pulses with the power of $1.0 \mathrm{~J}$ each looks safe, causes morphologic changes, restricted by the muscular layer and does not lead to the wall perforation.
\end{abstract}

Keywords: direct electropulse exposure, morphologic changes, urinary tracts

Cite as Gudkov AV, Boshchenko VS, Nedosekov VV. The morphologic changes in the wall of the human urinary tracts after direct electro pulse exposure: the in vitro research. Russian Open Medical Journal 2013; 2: 0108.

Correspondence to Dr Vyacheslav S. Boshchenko. Address: 2, Moskovskiy tract, Tomsk, 634050, Russia. Phone: +7 $3822417578,+79234261777$.

E-mail: vsbosh@mail.ru

\section{Introduction}

In the recent decade during the urolithiasis treatment the accent is made on the usage of modern low-invasive approaches like endourologic methods, mainly retrograde contact lithotripsy (RCL) [1-4]. In 2006 the electro pulse method which was offered as a new way of contact lithotripsy, which looked like splitting up the nephrolith by the exposure of the solid body by the electro pulses of nanosecond duration [5]. The radical difference of the new lithotripsy method from the highly effective, but unsafe because of high pulse power, electrohydraulic RCL and remote lithotripsy (RL) $[6,7]$, is exposure on the nephrolith surface, not on the fluid environment with the shockwave creation, affecting the stone. According to the experimental research data, carried out on the analogous nephrolith models, the change of solid body exposure pattern from mediated to direct allows to decrease power and number of electrical pulses, required for the effective lithotripsy, significantly [8]. The destruction of solid bodies with the same density by the means of electrohydraulic $R C L$ required exposure by multiple pulse series with the power of $1.85 \mathrm{~J}$, whereas in case of electro pulse RCL the single pulses with the power 0.1-0.45 J were enough; so, the minimal effective pulse power of electro pulse destruction method is 10 times lower that of the electrohydraulic RCL (0.1 and $1.0 \mathrm{~J}$ respectively). That allowed us to expect high level of clinical safety of the new method. At the same time, it is known that the higher the pulse power, the lower is crushing efficiency and time required for the stone destruction. In connection with that, for theelectro pulse RCL method clinical usage it is reasonable to estimate that maximum pulse power which is safe at the same time.

\section{Material and Methods}

In order to study the morphologic changes, happening in humanurinary tract wallafter direct electro pulse exposure, the surgical material obtained from the patients who had any operations on their pelvis, ureter or urinary bladder, leading to organ resection or removal. Thekidney pelvis, ureter or urinary bladder material, obtained interoperatively was divided into fragment right after extraction and put in the Petri dishes, filled with $0.9 \%$ sodium chlorides solution and single electric pulses of nanosecond duration were carried out directly on the mucous tunic.

The contact electric exposure was carried out by means of the probe of electro pulse "Urolit-105M" lithotriptor (Lithotech Medical, Israel; Medline Ltd, Russia). The pulse power during the exposure on kidney pelvis and ureter was $0.8 \mathrm{~J}$ (15 fragments of each organ) or $1.0 \mathrm{~J}$ (10 fragments of kidney pelvis and 18 ureter fragments), during the exposure on urinary bladder $-1.0 \mathrm{~J}$ in all cases ( 20 fragments). The number of electric pulses was varied in 
the range from 1 to 20 (1-3-5-10-20) for each localization. The fragment distribution of fragments depending on power and number of pulses summary table is introduced in the Table.

Table. The distribution of number of observed urinary excretion fragments with regard to power and number of used pulses

\begin{tabular}{|c|c|c|c|c|c|c|c|c|c|c|}
\hline \multirow{4}{*}{ Localization } & \multicolumn{10}{|c|}{ Parameter } \\
\hline & \multicolumn{5}{|c|}{ Power, $0.8 \mathrm{~J}$} & \multicolumn{5}{|c|}{ Power, $1.0 \mathrm{~J}$} \\
\hline & \multicolumn{5}{|c|}{ Pulse quantity } & \multicolumn{5}{|c|}{ Pulse quantity } \\
\hline & 1 & 3 & 5 & 10 & 20 & 1 & 3 & 5 & 10 & 20 \\
\hline Pelvis (n=25) & 3 & 3 & 3 & 3 & 3 & 2 & 2 & 2 & 2 & 2 \\
\hline Ureter $(n=33)$ & 3 & 3 & 3 & 3 & 3 & 3 & 3 & 4 & 4 & 4 \\
\hline $\begin{array}{l}\text { Urinary bladder } \\
(n=20)\end{array}$ & - & - & - & - & - & 4 & 4 & 4 & 4 & 4 \\
\hline
\end{tabular}

$\mathrm{n}-$ is the number of fragments used

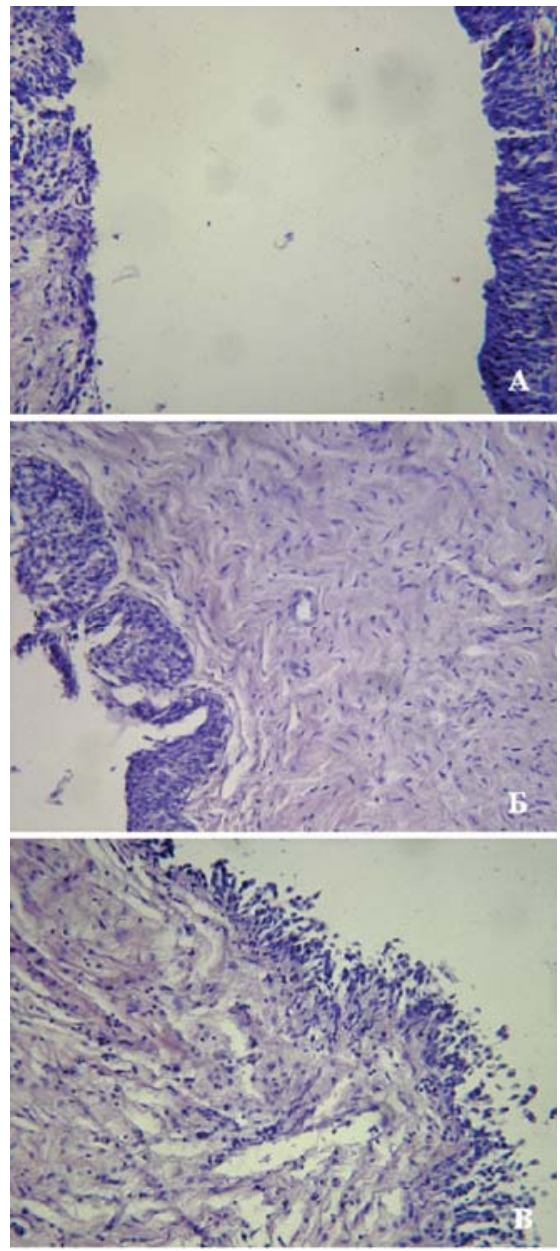

Figure 1. The Patient, 60 years old. The pelvis wall fragment after electro pulse exposure. Coloured by hematoxylin and eosin. Magnification 1x250:

A -5 pulses with the power of $0.8 \mathrm{~J}$. The mucous tunic deepithelization, integumentary epithelium desquamation right up to basal membrane in the exposure zone. The mucous tunic cover on the contralateral wall is saved, the layers stratification was not disrupted;

$5-20$ pulses with the power of $0.8 \mathrm{~J}$. Themucous tunicintegumentary epithelium epithelial layer cells discomplexation, surface epithelium cells partial desquamation. Submucous layers troma decondensation;

B - 5 pulses with the power of $1.0 \mathrm{~J}$. Discomplexation, fragmentation of mucous layer integumentary epitheliumepithelial layer cells, epithelium surface cells desquamation. Apparent edema, submucous layer stroma decondensation.

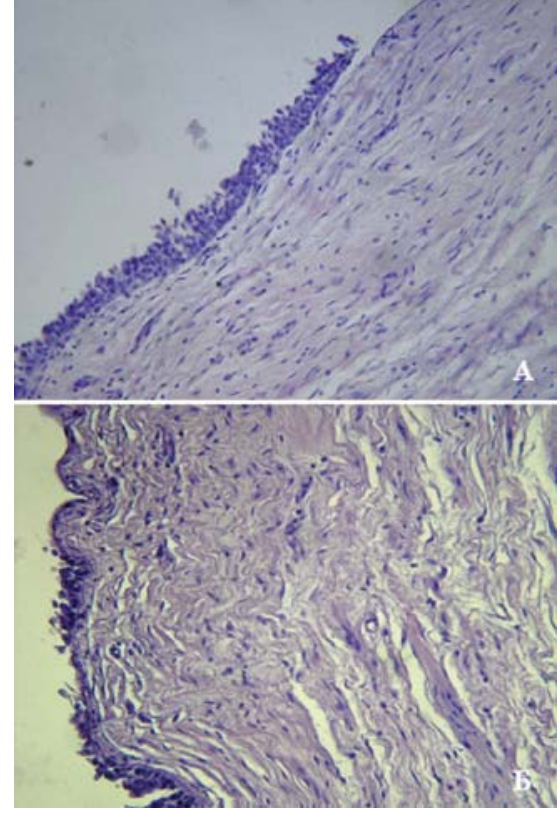

Figure 2. The Patient, 60 years old. The ureter wall fragment after electro pulse exposure. Coloured by hematoxylin and eosin. Magnitude 1x250:

A -3 pulses with the power of $0.8 \mathrm{~J}$. Partial desquamation of ureter mucous layer integumentary epithelium surface cells, moderate edema of sub mucous layer stroma;

5 -10 pulses with te power of $0.8 \mathrm{~J}$. Discomplexation, desquamation of surface ureter mucous tunic integumentary epithelium surface cells, in some areas right up to basic membrane, apparent edema of mucous layer stroma and sub mucous basis.

After finishing the electro pulse exposure, the fragments were put into marked vials and fixed into $10 \%$ neutralformalin solution. In the lab every tissue block was passed through the spirit battery, orientated on the wooden bar with its longitudinal (long) axis parallel to the latter's side, and then covered with paraffin according to conventional methods. The slices no more than 5-8 micrometers thick, made up from the paraffinized tissue block, were dyed with hematoxylin and eosin. The microscopy was conducted Micmed-2 microscope (Lomo, Saint-Petersburg, Russian Federation) with the magnification of 1:100 and 1:250.The pathomorphologist, conducting histologic research, did not have information about the pulse, any of the fragments was exposed, number and power.

\section{Results}

The kidney pelvis wall proved to be the most resistant to electro pulse exposure. During the exposure by 1-10 pulses with the power of $0.8 \mathrm{~J}$ (Figure $1 \mathrm{~A}$ ), epithelium desquamation without leaving thebasic membrane borders was detected, during the exposure by 20 pulses of the same power, the capillary dilatation was detected (Figure 15). Only exposure by 20 pulses with the maximum power of $1.0 \mathrm{~J}$ lead to changes out of urinary tract borders, such as singular hemorrhages in parapelvic cellular tissue (Figure 1B). The ureter wall was less resistant to direct electro pulse exposure than the pelvis wall.With the pulse power of $0.8 \mathrm{~J}$ and pulse number no more than 5 , the changes affected not only mucous tunic, but sub mucous layer as well, which was reflected as sub mucous layer edema (Figure 2). The exposure by 20 pulses of the maximum power $(1.0 \mathrm{~J})$ led to morphologic changes in the muscular layer, up to its dissociation. The urinary bladder wall 
proved to be the most vulnerable to direct electro pulse exposure. The electro pulse exposure, even by a singular pulse with the power of $1.0 \mathrm{~J}$ lead to sub mucous layer edema and collagenous fibres fragmentation (Figure $3 \mathrm{~A}$ ). The exposure by 5 consecutive pulses led to damaging not only epithelium andsub mucous base, the muscle layer as well (Figure $3 \mathrm{5}$ ), which reached the maximum in case of increase of pulse number to 20 , accompanied by vessels walls disruption (Figure $3 \mathrm{~B}$ ).

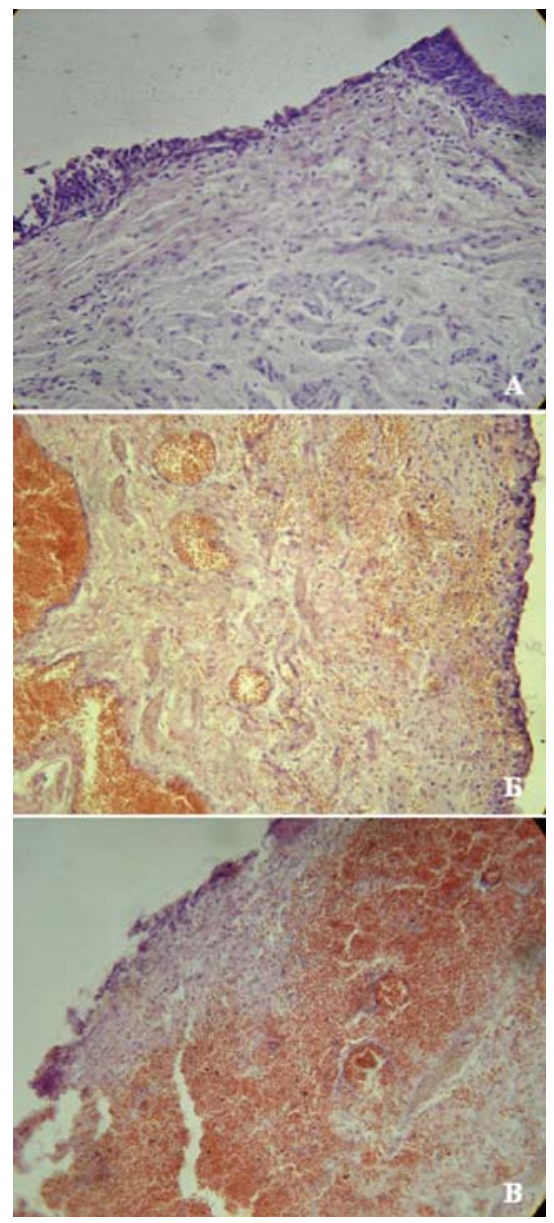

Figure 3. The Patient, $\mathbf{3 1}$ year old. The urinary bladder wall fragment after electro pulse exposure. Coloured by hematoxylin and eosin. Magnitude 1×250:

A - 1 pulse with the power of $1.0 \mathrm{~J}$. Discomplexation, fragmentation, desquamation of mucous layer epithelium right up to basal membrane in the exposure zone. Apparent edema, decondensation of mucous layer stroma and sub mucous basis, swelling, fragmentation of collagenous fibres;

$5-5$ pulses with the power of $1.0 \mathrm{~J}$. Full deepithelization of mucous layer, desquamation of the integumentary epithelium right up to basal membrane in the exposure zone. Apparent edema, decondensation of mucous tunic stroma and sub mucous basis, swelling, fragmentation of collagenous fibres.Acute venous hyperemia, erythrocytesstasis in the vessels lumen, haemorrhages in the sub mucous basis;

B -20 pulses with the power of $1.0 \mathrm{~J}$. The full deepithelization of mucous tunic, integumentary epithelium desquamation right up to basal membrane in the exposure zone. Apparent edema, decondensation of mucous tunic stroma and sub mucous basis, swelling, fragmentation of collagenous fibres. The vessels walls distruption, joint haemorrhages in the sub mucous basis and muscular basis together with urinary bladder wall imbibition by erythrocytes, destruction of bindweb and muscular bladder wall structures.

\section{Discussion}

In order to shift from experimental research toelectro pulse RCL clinical applicationwe had to identify optimal pulse power. On one hand, it had to be sufficient for maximal effective and quick nephrolith crushing. On the other hand, it had to avoid urinary tracts wall total damaging and perforation, even in case of electrode's end slipping-off from mucous tunic surface to the nephrolithduring the pulse exposure. Planning the research design, werelied on experimental and clinical facts, obtained earlier, notably: during the experiment the new electro pulse $\mathrm{RCL}$ method demonstrated high effectiveness in analogous sandcement mixture stone models destruction, including the samples consisting of $100 \%$ cement, during the singular pulse exposure with the power of 0.1 to $0.45 \mathrm{~J}[8,9]$. Such pulse power is comparable with the power of pulses, generated by the most safe RCL types, the pneumatic and laser ones $(0.1-0.14 \mathrm{~J}$ and $0.12-$ $0.5 \mathrm{~J}$, respectively) $[1,4,6,7,10]$.

It was ascertained during the numerous research that the higher the pulse power, the higher will be the crushing effectiveness and the lesser will be the time, needed for lithotripsy. The most efficient, although the most dangerous lithotripsy method - the electrohydraulic one, leading to ureter perforation (up to $17 \%$ ) quite often, has pulse power of 1.0 to 1.85 $J[1,8]$. Hence, it would be logical to assume that the safe level of pulse power in case of electro pulse RCL should stay in the range from from 0.1 to $1.0 \mathrm{~J}$.However, the closer the power lever to $1.0 \mathrm{~J}$, the higher will be the method efficiency. In thedog research, conducted earlier, it was demonstrated that directcontact electro pulse exposure by the pulses with the power of 0.1-0.7 $\mathrm{J}$ on the ureter and urinary bladder walleads to morphologic changes, not leaving the borders of muscular layer and considered completely safe [8]. At the same time, the exposure by the pulses with the power of 0.8 to $1.0 \mathrm{~J}$, causing changes in all in all ureter wall layers can be potentially dangerous and if the pulse power equals $1.0 \mathrm{~J}$ it may lead to wall perforation in individual cases. Since, the data obtained from animals and humans are not always absolutely concordant, in the current research we rejected pulse power range $0.1-0.7 \mathrm{~J}$, safe for dogs and studied morphological changes in human urinary tracts wall, occurring with pulse power range $0.8-$ $1.0 \mathrm{~J}$, relatively dangerous for dogs. As, the endoscopic contact exposure on undamaged human mucous tunic on this stage of method introduction could not even be considered for ethical reasons, we used pelvis, ureter and urinary bladder intact fragments, taken from the patients who had resection operations on their urinary tracts performed according to clinical evidence.

During this research we found out that direct electro pulse exposure, exposed on isolated pelvis, ureter and urinary bladder fragments by the pulses with the power of 0.8 to 1.0 Jin quantity from 1 to 20 did not lead to urinary system organs wall perforation. The kidney pelvis where the morphologic changes like epithelium desquamation, basal membrane disorganization, capillary paretic expansion and edema, did not leave sub mucous layer borders even after exposure by 10 pulses with the power of $1.0 \mathrm{~J}$ sentin one point, proved to be the most resistant to electro pulse exposure. In the fragments of ureter and urinary bladder analogous changes occurred after exposure by 5 pulses with the power of $1.0 \mathrm{~J}$ and after 10 pulses with the power of $1.0 \mathrm{~J}$ there were changes restricted by the muscular layer, but still not affecting adventitia. Such different kidney pelvis, ureter and urinary bladder resistance to electro pulse exposure can be explained by organs' anatomic difference and their walls structure 
patterns. The conditions designed by us exceeded the real threat which can be created by the method significantly, because it is impossible to assume that the probe will be regularly slipping-off the stone and that it will be slipping-off at the same point in case of pelvis and urinary bladder and very unlikely in case of lithotriptor, which is tightly fixed in ureter.The special feature of that research is that the result of any exposure - the electro pulse as well - on the living organ, having blood supply and innervation, and its fragment, even if it was removed, but not heaving blood supply already, can be slightly different.Nevertheless, the acquired data may be used as a basis, because electro pulse exposure was carried out directly in the operating room, and the time elapsed between new fragment extraction and pulses exposure did not exceed $1-2$ minutes, after that the material was preserved in formalin. However, considering relatively small electro pulse RCL usage period in case of urolithiasis in most of hospitals, it will be reasonable to start the method development from the pulse power no more than $0.7 \mathrm{~J}$, absolutely safe even in case of direct contact with the urinary tracts walls [9]. Only, after obtaining enough clinical experience of manipulation with lithotriptor probe and stop-filters, helping to fix the stone during the lithotripsy process, the exposure power can be increased to $1.0 \mathrm{~J}$.

\section{Conclusion}

1) The contact electro pulse exposure on pelvis, urinary bladder and ureter mucous tunic by 10 pulses with the pulse power of $1.0 \mathrm{~J}$ each is safe and does not cause morphologic changes out of muscular layer borders.

2) The contact electro pulse exposure by the pulses with the power of $0.8 \mathrm{~J}$ to $1.0 \mathrm{~J}$ from 1 to 20 pulses does not lead to urinary excretion system organs' walls.

Conflict of interest: none declared

\section{Reference}

1. Martov AG, Safarov RM, Guschchin BL, Kudrjavcev JuV. Comparative effectiveness and safety of usage of different contact lithotripter. The ruling plenum of Russian urologist society. Moskow, Russia, 1998: 312313. [Text in Russian]

2. Pearle MS, Pierce HL, Miller GL, Summa JA, Mutz JM, Petty BA, et al. Optimal method of urgent decompression of the collecting system for obstruction and infection due to ureteral calculi. J Urol 1998; 160(4): 1260-4. (PMID: 9751331)

3. Jung $P$, Wolff JM, Mattelaer $P$, Jakse $G$. Role of lasertripsy in the management of ureteral calculi: experience with alexandrite laser system in 232 patients. J Endourol 1996; 10(4): 345-8. (PMID: 8872732)

4. Pearle MS, Sech SM, Cobb CG, Riley JR, Clark PJ, Preminger GM, et al. Safety and efficacy of the Alexandrite laser for the treatment of renal and ureteral calculi. Urology 1998; 51(1): 33-8. (PMID: 9457285)

5. Usov AV, Semkin BV, Zinov'ev NT. Transitional processes in electro pulse technologies usage. Nauka Publ, Saint-Petersburg, Russia, 2000. [Text in Russian]

6. Boschenko VS, Gudkov AV, Tilashov EM, Afonin VY. The results of usage of new contact electro pulse lithotriptor "Urolit" to destroy the ureter and urinary bladder lithotripters. The Bashkortostan medical bulletin 2007; (2): 34-35. [Article in Russian]

7. Bierkens AF, Hendrikx AJ, De La Rosette JJ, Stultiens GN, Beerlage HP, Arends AJ, Debruyne FM. Treatment of mid- and lower ureteric calculi: extracorporeal shock-wave lithotripsy vs laser ureteroscopy. A comparison of costs, morbidity and effectiveness. Br J Urol 1998; 81(1): 31-5. (PMID: 9467473)
8. Afonin VY. Electropulse contact destruction of the ureter and urinary bladder lithotriptors. Candidates of medical science dissertation. Saratov, Russia, 2009.

9. Gudkov AV, Boshchenko VS, Afonin Vla. Contact electroimpulse lithotripsy. Urologiia 2009; (2): 32-7. (PMID: 19530326) [Article in Russian]

10. Sofer M, Watterson JD, Wollin TA, Nott L, Razvi H, Denstedt JD. Holmium:YAG laser lithotripsy for upper urinary tract calculi in 598 patients. J Urol 2002; 167(1): 31-4. (PMID: 11743269)

\section{Authors:}

A.V. Gudkov - MD, D.Sc., Professor, Head of Department of Urology, Siberian State Medical University, Tomsk, Russia;

Vyacheslav S. Boshchenko - MD, PhD, Assistant Professor, Department of Urology, Siberian State Medical University, Tomsk, Russia;

V.V. Nedosekov - MD, PhD, Assistant Professor, Department of Pathological Anatomy, Siberian State Medical University, Tomsk, Russia. 